\title{
Linx
}

Revue des linguistes de l'université Paris X Nanterre

48 | 2003

Approches syntaxiques contemporaines

\section{Carnie, Andrew (2002) Syntax. A Generative}

Introduction

Oxford, Blackwell Publishing, 390 pages, 55 livres

Danielle Leeman

\section{CpenEdition}

\section{Journals}

Édition électronique

URL : http://journals.openedition.org/linx/234

DOI : $10.4000 /$ linx.234

ISSN : 2118-9692

\section{Éditeur}

Presses universitaires de Paris Nanterre

Édition imprimée

Date de publication : 1 juin 2003

Pagination : 164

ISBN : 0246-8743

ISSN : 0246-8743

\section{Référence électronique}

Danielle Leeman, «Carnie, Andrew (2002) Syntax. A Generative Introduction», Linx [En ligne], 48 | 2003,

mis en ligne le 01 octobre 2003, consulté le 22 septembre 2020. URL : http://journals.openedition.org/ linx/234; DOI : https://doi.org/10.4000/linx.234 


\section{Comptes rendus par Danielle Leeman}

\section{Carnie, Andrew (2002) Syntax. A Generative Introduction, Oxford, Blackwell Publishing, 390 p., $55 £$}

Voilà un manuel d'initiation progressive et extrêmement pédagogique à ce que l'on a coutume d'appeler «la grammaire générative» (dans sa période préminimaliste ${ }^{1}$ ), pour ce qui concerne sa composante syntaxique. Si nos étudiants achetaient des livres (en Sciences du langage) et s'ils acceptaient de lire de l'anglais (dès les premières années de leurs études universitaires), cet ouvrage pourrait constituer le support idéal de leurs premiers pas en linguistique.

La première partie en effet présente, très simplement, les grands soubassements de la théorie (le rapport au «cognitif», la notion de "modèle », les différents types d'adéquation d'une grammaire, la démarche scientifique) et les concepts utiles au raisonnement syntaxique (parties du discours, relations structurales, tests de constituance, arbre, règle). La seconde partie expose très clairement le mode de représentation de la structure de la phrase et de ses constituants (la théorie X', les rôles thématiques, le statut du lexique). La troisième partie introduit les transformations de mouvement, et les notions de "montée », «contrôle », «catégorie vide» en en montrant la pertinence et la rentabilité sur des exemples extraits de différentes langues et précisément commentés. Une quatrième partie présente deux «théories alternatives»: la Grammaire Lexicale Fonctionnelle et la Grammaire Syntagmatique Guidée par les Têtes².

Chacun des chapitres, qui mène vraiment le lecteur pas à pas, prévoit ses difficultés et attire l'attention sur les erreurs à ne pas commettre (l'auteur est manifestement un enseignant qui observe attentivement ses étudiants), se clôt par une brève conclusion synthétisant ce qui est à retenir, assortie d'un glossaire rappelant la définition des principales notions introduites, de quelques références complémentaires brièvement commentées, et de "petits problèmes » qui vont du simple exercice de vérification au sujet de réflexion sur les langues les plus variées (leur corrigé, comme tout autre renseignement, peut être obtenu sur le site de l'éditeur).

On ne peut qu'engager vivement les bibliothèques à commander l'ouvrage et les responsables pédagogiques à en recommander la lecture active à leurs étudiants 3 .

\footnotetext{
${ }^{1}$ Sur l'état contemporain de la théorie, dit «Minimalisme», cf. dans le présent numéro de Linx l'article de Patrick Bellier.

${ }^{2}$ Sur la première, voir: Abeillé, A. (1993) Les nowvelles syntaxes, Paris, Colin et le compte rendu ciaprès ; sur la seconde, cf. A. Abeillé (op. cit.) et Abeillé, A. (2002) Une grammaire électronique du français, Paris, CNRS, ainsi que, dans le présent numéro de Linx, l'article de Marianne Desmets et al.

${ }^{3}$ Citons aussi, dans la période récente, l'excellente «introduction à la syntaxe générative » de A. Zribi-Hertz: Zribi-Hertz, A. (1996) L'anaphore et les pronoms, Lille, Presses universitaires du Septentrion, 280 p. - toutefois centrée sur une thématique (comme l'indique le titre) et donc sur l'une des composantes de la grammaire (la théorie du liage). Mentionnons également en plus généraliste et moins soigneusement didactique : Moeschler, J. et Auchlin, A. (1997) Introduction à la linguistique contemporaine, Paris, Colin, coll. « cursus », 192 p., et Pollock, J.-Y. (1997) Langage et cognition. Introduction au programme minimaliste de la grammaire générative, Paris, PUF, 242 p., ou, en plus
} 